\title{
Posterior Analysis of State Space Model with Spherical Symmetricity
}

\author{
Ranjita Pandey \\ Department of Statistics, University of Delhi, Delhi 110007, India \\ Correspondence should be addressed to Ranjita Pandey; ranjitapandeyll1@gmail.com
}

Received 28 June 2015; Revised 15 October 2015; Accepted 18 October 2015

Academic Editor: Shein-chung Chow

Copyright ( 2015 Ranjita Pandey. This is an open access article distributed under the Creative Commons Attribution License, which permits unrestricted use, distribution, and reproduction in any medium, provided the original work is properly cited.

\begin{abstract}
The present work investigates state space model with nonnormal disturbances when the deviation from normality has been observed only with respect to kurtosis and the distribution of disturbances continues to follow a symmetric family of distributions. Spherically symmetric distribution is used to approximate behavior of symmetric nonnormal disturbances for discrete time series. The conditional posterior densities of the involved parameters are derived, which are further utilized in Gibbs sampler scheme for estimating the marginal posterior densities. The state space model with disturbances following multivariate- $t$ distribution, which is a particular case of spherically symmetric distribution, is discussed.
\end{abstract}

\section{Introduction}

Often in the analysis of various time series dynamic or state space models, the distribution of disturbances is assumed to be normal. However, the assumption of normal disturbances may not be satisfied in many practical applications of these models. For example, deviations from normality have been observed in stock price data where behavior of disturbances appears to be leptokurtic; see, for example, Kendall [1] and Fama [2]. Praetz [3] suggested scaled- $t$ distribution to explain leptokurtic behavior of stock price data. If the tails of the distribution are flatter than the normal distribution, the multivariate- $t$ distribution may provide a more realistic model for the disturbances. Zellner [4] revealed that dependent but uncorrelated responses could be analyzed using a student- $t$ model. Fraser [5] demonstrated the robustness of the student- $t$ family as opposed to the normal distribution based on empirical studies and further suggested that a student- $t$ model holds equally good for normal model responses. Another advantage of student- $t$ family is that it handles outliers easily; see Sutradhar and Ali [6]. Student- $t$ model is also advocated by Haq and Khan [7], Khan and Haq [8], and Chib et al. [9]. Prucha and Kelejian [10] observed that normal model based analysis is heavily influenced by extreme observations and deviations from assumptions and ignores sample information beyond the first two moments. Robustness for Bayes prediction under multivariate- $t$ case has also been studied by Chib et al. [11], which is a particular member of the spherically symmetric family. Jammalamadaka et al. [12] have, however, shown that predictive inferences are completely uninfluenced by departure from the normality assumption in the direction of the spherically symmetric family. Properties of the spherical distribution have been studied by Kelker [13] and Chmielewski [14]. In carrying out the Bayesian analysis of various dynamic models involving multidimensional parameter vector, it is often difficult to derive explicit expressions for the marginal posterior distributions of various parameters. In such circumstances, Markov Chain Monte Carlo (MCMC) technique, also known as Gibbs sampler, provides a useful way for estimating the marginal posterior densities. Klock and Van Dijk [15], Naylor and Smith [16], and Geweke [1719] applied numerical MCMC for the Bayesian analysis of various time series models. Tiwari et al. [20] considered a general state space or linear dynamic model with normal disturbances and carried out the Bayesian analysis of the model using Gibbs sampler. In the present exposition, the assumption of normality of disturbances is relaxed and it is assumed that the disturbances follow distribution belonging to the family of spherically symmetric distributions. 


\section{Model and Assumptions}

The following form of general state space (or linear dynamic model) is considered:

$$
\begin{aligned}
& y_{t}=x_{t}^{\prime} \theta_{t}+\varepsilon_{t}, \\
& \theta_{t}=G_{t} \theta_{t-1}+v_{t},
\end{aligned}
$$

$$
(t=1,2, \ldots, T),
$$

where $y_{t}$ is the observation on the response variable, $x_{t}$ is a $p \times 1$ regression vector, $\theta_{t}$ is a $p \times 1$ vector of parameters generated by the first-order autoregressive model in (1), and $G_{t}$ is a known $p \times p$ transition matrix. The initial value of parameter at $t=0$, that is, $\theta_{0}$, is assumed to be unknown with its uncertainty expressed by the following normal distribution $\theta_{0}$ । $\lambda \sim N_{p}\left(m_{0}, \lambda^{-1} \Sigma_{0}\right), \lambda \sim G\left(a_{0} / 2, b_{0} / 2\right)$, where $G\left(a_{0} / 2, b_{0} / 2\right)$ denotes a Gamma distribution with density function defined by $p(\lambda)=\left(\left(b_{0} / 2\right)^{a_{0} / 2} / \Gamma\left(a_{0} / 2\right)\right) \lambda^{a_{0} / 2-1} e^{-\left(b_{0} / 2\right) \lambda}$ and $\Sigma_{0}$ is a $p \times p$ positive definite, symmetric matrix. The hyperparameters $a_{0}$, $b_{0}, m_{0}$, and $\Sigma_{0}$ are assumed to be known. The errors $\varepsilon_{t}$ and $v_{s}$ are assumed to be independent for all $t$ and $s$. The joint distribution of $\varepsilon=\left(\varepsilon_{1}, \ldots, \varepsilon_{T}\right)^{\prime}$ is assumed to be spherically symmetric distribution with joint density function given by

$$
\begin{aligned}
& f(\varepsilon)=\int_{0}^{\infty} \frac{\lambda^{T / 2}}{(2 \pi)^{T / 2} \sigma_{0}^{T} \psi^{T}(\zeta)} \exp \left\{-\frac{\lambda}{2 \sigma_{0}^{2} \psi^{2}(\zeta)}\right. \\
& \left.. \sum_{t=1}^{T} \varepsilon_{t}^{2}\right\} d G(\zeta),
\end{aligned}
$$

where $\Psi(\zeta)$ is a positive measurable function and pdf and cdf of $\zeta$ are represented by $g(\zeta)$ and $G(\zeta)$, respectively. We further assume that the errors $v_{t}$ follow the normal distribution with mean 0 and variance $\lambda^{-1} \Sigma$, where $\Sigma$ is a $p \times p$ positive definite symmetric known matrix.

Thus, $E\left[\theta_{t} \mid \theta_{t-1}, \lambda\right]=G_{t} \theta_{t-1}$ and $V\left[\theta_{t} \mid \theta_{t-1}, \lambda\right]=\lambda^{-1} \Sigma$. Thus, $\theta_{t} \mid \theta_{t-1} \sim N_{p}\left(G_{t} \theta_{t-1}, \lambda^{-1} \Sigma\right)$.

\section{Conditional Posterior Densities}

The density function of the observation vector $y=\left(y_{1}\right.$, $\left.\ldots, y_{T}\right)^{\prime}$ is given by

$$
\begin{aligned}
& f(\underset{\sim}{y} \mid \underset{\sim}{\theta}, \lambda)=\int_{0}^{\infty} \frac{\lambda^{T / 2}}{(2 \pi)^{T / 2} \sigma_{0}^{T} \psi^{T}(\zeta)} \exp \left\{-\frac{\lambda}{2 \sigma_{0}^{2} \psi^{2}(\zeta)}\right. \\
& \left.\quad \cdot \sum_{t=1}^{T}\left(y_{t}-x_{t}^{\prime} \theta_{t}\right)^{2}\right\} d G(\zeta) .
\end{aligned}
$$

In other words, the conditional distribution of $y$ given $\zeta$ is normal with mean $\left(x_{1}^{\prime} \theta_{1}, \ldots, x_{T}^{\prime} \theta_{T}\right)^{\prime}$ and covariance matrix $\left(\sigma_{0}^{2} / \lambda\right) \psi^{2}(\zeta) I_{T}$. Since it is difficult to derive the explicit expressions for the marginal posterior densities of the parameters of the model, Gibbs sampler scheme can be used for estimating the marginal posterior densities. The conditional posterior densities of different parameters derived in this section can be utilised in Gibbs sampler scheme. The following joint distribution of $\left(y,\left\{\theta_{t}\right\}_{t=0}^{T}, \lambda\right)$ given $\zeta$ is obtained as follows:

$$
\begin{aligned}
& f\left(\underset{\sim}{y},\left\{\theta_{t}\right\}_{t=0}^{T}, \lambda \mid \zeta\right) \propto \frac{\lambda^{T / 2}}{\{\psi(\zeta)\}^{T}} \\
& \cdot \exp \left\{-\frac{\lambda}{2 \sigma_{o}^{2} \psi^{2}(\zeta)} \sum_{t=1}^{T}\left(y_{t}-x_{t}^{\prime} \theta_{t}\right)^{2} \lambda^{(T p / 2)^{2}} \lambda^{a_{0} / 2-1}\right. \\
& \cdot \exp \left\{-\frac{b_{0}}{2} \lambda\right\} \\
& \cdot \exp \left\{-\frac{\lambda}{2} \sum_{t=1}^{T}\left(\theta_{t}-G_{t} \theta_{t-1}\right)^{\prime} \Sigma^{-1}\left(\theta_{t}-G_{t} \theta_{t-1}\right)\right\} \lambda^{p / 2} \\
& \cdot \exp \left\{-\frac{\lambda}{2}\left(\theta_{0}-m_{0}\right)^{\prime} \Sigma_{0}^{-1}\left(\theta_{0}-m_{0}\right)\right\} .
\end{aligned}
$$

Let us write, $K_{t}(\zeta)=\lambda^{p / 2} /(2 \pi)^{p / 2}\left|B_{t}(\zeta)\right|^{1 / 2} ; t=0,1, \ldots, T$, $\theta^{(s)}=\left\{\theta_{t}\right\}_{t=0}^{T}$ for $t \neq s$

$$
\begin{gathered}
b_{t}(\zeta)= \begin{cases}G_{1}^{\prime} \Sigma^{-1} \theta_{1}+\Sigma_{0}^{-1} m_{0}, & \text { for } t=0 \\
\frac{1}{\sigma_{0}^{2} \psi^{2}(\zeta)} x_{t} y_{t}+\Sigma^{-1} G_{t} \theta_{t-1}+G_{t+1}^{\prime} \Sigma^{-1} \theta_{t+1}, & \text { for } t=1,2, \ldots T-1 \\
\frac{1}{\sigma_{0}^{2} \psi^{2}(\zeta)} x_{T} y_{T}+\Sigma^{-1} G_{T} \theta_{T-1}, & \text { for } t=T,\end{cases} \\
B_{t}(\zeta)^{-1}= \begin{cases}\Sigma_{0}^{-1}+G_{1}^{\prime} \Sigma^{-1} G_{1}, & \text { for } t=0 \\
\frac{1}{\sigma_{0}^{2} \psi^{2}(\zeta)} x_{t} x_{t}^{\prime}+\Sigma^{-1}+G_{t+1}^{\prime} \Sigma^{-1} G_{t+1}, & \text { for } t=1,2, \ldots T-1 \\
\frac{1}{\sigma_{0}^{2} \psi^{2}(\zeta)} x_{T} x_{T}^{\prime}+\Sigma^{-1}, & \text { for } t=T .\end{cases}
\end{gathered}
$$

Theorem 1. The conditional posterior density of $\theta_{t}$ is given by

$$
f^{*}\left(\theta_{t} \mid \underset{\sim}{y}, \lambda\right)=\int_{0}^{\infty} K_{t}(\zeta) \exp \left\{-\frac{\lambda}{2}\left(\theta_{t}-B_{t}(\zeta) b_{t}(\zeta)\right)^{\prime}\right.
$$

$$
\left.\cdot B_{t}(\zeta)^{-1}\left(\theta_{t}-B_{t}(\zeta) b_{t}(\zeta)\right)\right\} g(\zeta) d \zeta
$$$$
\text { for } t=0,1, \ldots, T-1, T \text {. }
$$ 
Proof. For $t=0$, using expression (4), we get

$$
\begin{aligned}
& f^{*}\left(\theta_{0} \mid \underset{\sim}{\underset{\gamma}{y}}, \lambda, \zeta\right) \propto \exp \left[-\frac{\lambda}{2}\left\{\theta_{0}^{\prime}\left(\Sigma_{0}^{\prime}+G_{1}^{\prime} \Sigma^{\prime-1} G_{1}\right) \theta_{0}\right.\right. \\
& \left.\left.\quad-2\left(\theta_{1}^{\prime} \Sigma^{-1} G_{1}+m_{0}^{\prime} \Sigma_{0}^{-1}\right) \theta_{0}\right\}\right]
\end{aligned}
$$

or

$$
\begin{aligned}
f^{*} & \left(\theta_{0} \mid \underset{\sim}{y}, \lambda, \zeta\right)=K_{0}(\zeta) \exp \left[-\frac{\lambda}{2}\left\{\left(\theta_{0}-B_{0}(\zeta) b_{0}(\zeta)\right)^{\prime}\right.\right. \\
\cdot & \left.\left.B_{0}(\zeta)^{-1}\left(\theta_{0}-B_{0}(\zeta) b_{0}(\zeta)\right)\right\}\right]
\end{aligned}
$$

where $K_{0}(\zeta)$ is the normalizing constant. For obtaining the value of $K_{0}(\zeta)$, we have

$$
K_{0}(\zeta)^{-1}=\int_{R^{p}} \exp \left[-\frac{\lambda}{2}\left\{\left(\theta_{0}-B_{0}(\zeta) b_{0}(\zeta)\right)^{\prime} B_{0}(\zeta)^{-1}\left(\theta_{0}-B_{0}(\zeta) b_{0}(\zeta)\right)\right\}\right] d \theta_{0}=\frac{(2 \pi)^{p / 2}\left|B_{0}(\zeta)\right|^{1 / 2}}{\lambda^{p / 2}}
$$

for $t=s$, where $s=1,2, \ldots, T-1$, and using expression (4) we get

$$
\begin{aligned}
& f^{*}\left(\theta_{s} \mid \underset{\sim}{y}, \lambda, \theta^{(s)}, \zeta\right) \\
& \propto \exp \left[-\frac{\lambda}{2}\left\{\left\{\theta_{s}^{\prime}\left(\frac{1}{\sigma_{0}^{2} \psi^{2}(\zeta)} x_{s} x_{s}^{\prime}+\Sigma^{-1}+G_{s+1}^{\prime} \Sigma^{-1} G_{s+1}\right)\right.\right.\right. \\
& \left.\cdot \theta_{s}\right\}-2 \theta_{s}^{\prime}\left\{\frac{1}{\sigma_{0}^{2} \psi^{2}(\zeta)} x_{s} y_{s}+\Sigma^{-1} G_{s} \theta_{s-1}\right. \\
& \left.\left.\left.+G_{s+1}^{\prime} \Sigma^{-1} \theta_{s+1}\right\}\right\}\right] \propto \exp \left[-\frac{\lambda}{2}\left\{\theta_{s}^{\prime} B_{s}(\zeta)^{-1} \theta_{s}-2 b_{s}(\zeta) \theta_{s}\right.\right. \\
& \left.\left.+b_{s}(\zeta)^{\prime} B_{s}(\zeta) b_{s}(\zeta)\right\}\right], \\
& f^{*}\left(\theta_{s} \mid \underset{\sim}{y}, \lambda, \theta^{(s)}, \zeta\right)=K_{s}(\zeta) \exp \left[-\frac{\lambda}{2}\left\{\left(\theta_{s}-B_{s}(\zeta) b_{s}(\zeta)\right)^{\prime}\right.\right. \\
& \left.\left.\quad \cdot B_{s}(\zeta)^{-1}\left(\theta_{s}-B_{s}(\zeta) b_{s}(\zeta)\right)\right\}\right],
\end{aligned}
$$

where the normalizing constant $K_{s}(\zeta)$ is obtained as

$$
\begin{gathered}
K_{s}(\zeta)^{-1}=\int_{R^{p}} \exp \left[-\frac{\lambda}{2}\left\{\left(\theta_{s}-B_{s}(\zeta) b_{s}(\zeta)\right)^{\prime} B_{s}(\zeta)^{-1}\right.\right. \\
\left.\left.\cdot\left(\theta_{s}-B_{s}(\zeta) b_{s}(\zeta)\right)\right\}\right]=\frac{(2 \pi)^{p / 2}\left|B_{s}(\zeta)\right|^{1 / 2}}{\lambda^{p / 2}}
\end{gathered}
$$

for $t=T$, and utilizing expression (4), we get

$$
\begin{aligned}
f^{*} & \left(\theta_{T} \mid \underset{\sim}{\underset{y}{y}}, \lambda,\left\{\theta_{t}\right\}_{t=0}^{T-1}, \zeta\right) \\
& \propto \exp \left[-\frac{\lambda}{2}\left\{\theta_{T}^{\prime}\left(\frac{1}{\sigma_{0}^{2} \psi^{2}(\zeta)} x_{T} x_{T}^{\prime}+\Sigma^{-1}\right)^{-1} \theta_{T}\right.\right. \\
& \left.\left.-2 \theta_{T}^{\prime}\left(\frac{1}{\sigma_{0}^{2} \psi^{2}(\zeta)} x_{T} y_{T}^{\prime}+\Sigma^{-1} G_{T} \theta_{T-1}\right)\right\}\right] \\
& \propto \exp \left[-\frac{\lambda}{2}\left\{\theta_{T}^{\prime} B_{T}(\zeta)^{-1} \theta_{T}-2 \theta_{T}^{\prime} b_{T}(\zeta)+b_{T}(\zeta)^{\prime}\right.\right. \\
\cdot & \left.\left.B_{T}(\zeta) b_{T}(\zeta)\right\}\right] \propto \exp \left[-\frac{\lambda}{2}\left\{\left(\theta_{T}-B_{T}(\zeta) b_{T}(\zeta)\right)^{\prime}\right.\right. \\
\cdot & \left.\left.B_{T}(\zeta)^{-1}\left(\theta_{T}-B_{T}(\zeta) b_{T}(\zeta)\right)\right\}\right]
\end{aligned}
$$

or

$$
\begin{aligned}
& f^{*}\left(\theta_{T} \mid \underset{\sim}{y}, \lambda,\left\{\theta_{t}\right\}_{t=0}^{T-1}, \zeta\right)=K_{T}(\zeta) \\
& \cdot \exp \left[-\frac{\lambda}{2}\left\{\left(\theta_{T}-B_{T}(\zeta) b_{T}(\zeta)\right)^{\prime} B_{T}(\zeta)^{-1}\right.\right. \\
&\left.\left.\cdot\left(\theta_{T}-B_{T}(\zeta) b_{T}(\zeta)\right)\right\}\right]
\end{aligned}
$$

where $K_{T}(\zeta)$ is the normalizing constant given by

$$
K_{T}(\zeta)^{-1}=\int_{R^{p}} \exp \left[-\frac{\lambda}{2}\left\{\left(\theta_{T}-B_{T}(\zeta) b_{T}(\zeta)\right)^{\prime} B_{T}(\zeta)^{-1}\left(\theta_{T}-B_{T}(\zeta) b_{T}(\zeta)\right)\right\}\right] d \theta_{T}=\frac{(2 \pi)^{p / 2}\left|B_{T}\right|^{1 / 2}}{\lambda^{p / 2}}
$$

Remark 2. It is interesting to observe from the above theorems that the conditional posterior density of $\theta_{0}$ given $\left(\left\{\theta_{t}\right\}_{t=1}^{T}, \lambda\right)$ depends only upon $\left(\theta_{1}, \lambda\right)$ and the conditional posterior density of $\theta_{s}(s=1, \ldots, T-1)$ given $\left(\left\{\theta_{t}\right\}_{t=0}^{T}(t \neq\right.$ $s), \lambda)$ depends only upon $\left(\theta_{s-1}, \theta_{s+1}, \lambda\right)$ whereas the conditional posterior density of $\theta_{T}$ given $\left(\left\{\theta_{t}\right\}_{t=0}^{T-1}, \lambda\right)$ depends only 
upon $\left(\theta_{T-1}, \lambda\right)$. Therefore, the conditional posterior densities for $\theta_{s}(s=0,1, \ldots, T)$ depend upon (one or two) values of $\theta_{t}$ adjacent to $\theta_{s}$ and $\lambda$.

Now, we define

$$
\begin{aligned}
a^{*}= & T p+T+p+a_{0}=(T+1)(p+1)+a_{0}-1, \\
b^{*}(\zeta)= & \frac{1}{\sigma_{0}^{2} \psi^{2}(\zeta)} \sum_{t=1}^{T}\left(y_{t}-x_{t}^{\prime} \theta_{t}\right)^{2} \\
& +\sum_{t=1}^{T}\left(\theta_{t}-G_{t} \theta_{t-1}\right)^{\prime} \Sigma^{-1}\left(\theta_{t}-G_{t} \theta_{t-1}\right) \\
& +\left(\theta_{0}-m_{0}\right)^{\prime} \Sigma_{0}^{-1}\left(\theta_{0}-m_{0}\right)+b_{0}, \\
C(\zeta)= & \frac{\left[b^{*}(\zeta) / 2\right]^{a^{*} / 2}}{\Gamma\left(a^{*} / 2\right)} .
\end{aligned}
$$

Theorem 3. The conditional posterior of $\lambda$ is obtained as

$$
\begin{aligned}
f^{*} & \left(\lambda \mid\left\{\theta_{t}\right\}_{t=0}^{T}, \underset{\sim}{y}\right) \\
& =\int_{0}^{\infty} C(\zeta) \lambda^{a^{*} / 2-1} \exp \left\{-\frac{\lambda}{2} b^{*}(\zeta)\right\} g(\zeta) d \zeta .
\end{aligned}
$$

Proof. Following (4), we observe that

$$
\begin{gathered}
f^{*}\left(\lambda \mid\left\{\theta_{t}\right\}_{t=0}^{T}, \underset{\sim}{\underset{ }{y}}, \zeta\right) \propto \lambda^{(1 / 2)\left(T+p+T p+a_{0}\right)-1} \\
\cdot \exp \left[-\frac{\lambda}{2}\left\{\frac{1}{\sigma_{0}^{2} \psi^{2}(\zeta)} \sum_{t=1}^{T}\left(y_{t}-x_{t}^{\prime} \theta_{t}\right)^{2}\right.\right. \\
+\sum_{t=1}^{T}\left(\theta_{t}-G_{t} \theta_{t-1}\right)^{\prime} \Sigma^{-1}\left(\theta_{t}-G_{t} \theta_{t-1}\right) \\
\left.\left.+\left(\theta_{0}-m_{0}\right)^{\prime} \Sigma_{0}^{-1}\left(\theta_{0}-m_{0}\right)+b_{0}\right\}\right] .
\end{gathered}
$$

The normalizing constant is given by

$$
\begin{aligned}
C(\zeta)^{-1} & =\int_{0}^{\infty} f^{*}\left(\lambda \mid\left\{\theta_{t}\right\}_{t=0}^{T}, \underset{\sim}{y}, \zeta\right) d \lambda \\
& =\frac{\Gamma\left(a^{*} / 2\right)}{\left\{b^{*}(\zeta) / 2\right\}^{a^{*} / 2}} .
\end{aligned}
$$

Remark 4. The above derived expressions for the conditional posterior densities of $\theta_{0}, \theta_{s}(s=1, \ldots, T-1), \theta_{T}$, and $\lambda$ involve integration with respect to $d G(\zeta)$. Sometimes, it may not be possible to obtain these integrals in neat form. In such situation, we consider $\zeta$ as a parameter with prior density function $g(\zeta)$ and derive the conditional posterior density functions of $\theta_{s}$ given $\left(\theta^{(s)}, \lambda, \zeta\right), \lambda$ given $\left(\left\{\theta_{t}\right\}_{t=0}^{T}, \zeta\right)$, and $\zeta$ given $\left(\left\{\theta_{t}\right\}_{t=0}^{T}, \lambda\right)$. The posterior conditional density functions of $\theta_{t}$ and $\lambda$ can be straightaway obtained as follows:

$$
\begin{aligned}
& f^{*}\left(\theta_{s} \mid \underset{\sim}{y}, \theta^{(s)}, \lambda, \zeta\right)=K_{s}(\zeta) \\
& \cdot \exp \left[-\frac{\lambda}{2}\left\{\left(\theta_{s}-B_{s}(\zeta) b_{s}(\zeta)\right)^{\prime} B_{s}(\zeta)^{-1}\right.\right. \\
& \cdot\left.\left.\left(\theta_{s}-B_{s}(\zeta) b_{s}(\zeta)\right)\right\}\right] \quad \text { where }(s=0,1, \ldots, T), \\
& f^{*}\left(\lambda \mid \underset{\sim}{y},\left\{\theta_{t}\right\}_{t=0}^{T}, \zeta\right)=C(\zeta) \exp \left(-\frac{\lambda}{2} b^{*}(\zeta)\right) \lambda^{\left(a^{*} / 2-1\right)} .
\end{aligned}
$$

Hence, the conditional posterior density of $\theta_{s}$ is normal $N_{p}\left(B_{s}(\zeta) b_{s}(\zeta), \lambda^{-1} B_{s}(\zeta)\right)$ and conditional posterior density of $\lambda$ is gamma.

Theorem 5. The conditional posterior density of $\zeta$ given $\left(\left\{\theta_{t}\right\}_{t=0}^{T}, \lambda\right)$ is given by

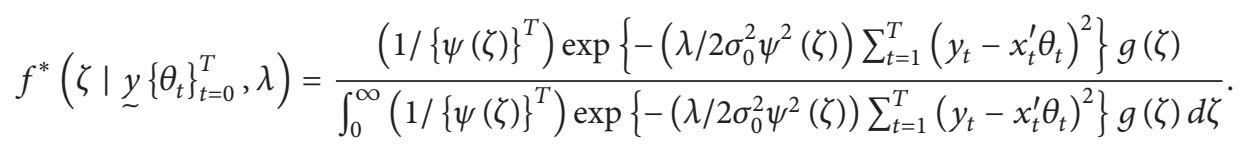

Proof. The joint density function of $\left(y,\left\{\theta_{t}\right\}_{t=0}^{T}, \lambda, \zeta\right)$ is given by

$$
\begin{aligned}
& f\left(\underset{\sim}{y},\left\{\theta_{t}\right\}_{t=0}^{T}, \lambda, \zeta\right) \propto \frac{\lambda^{T / 2}}{\{\psi(\zeta)\}^{T}} \\
& \cdot \exp \left\{-\frac{\lambda}{2 \sigma_{0}^{2} \psi^{2}(\zeta)} \sum_{t=1}^{T}\left(y_{t}-x_{t}^{\prime} \theta_{t}\right)^{2}\right\} \lambda^{T p / 2} \\
& \cdot \exp \left\{-\frac{\lambda}{2} \sum_{t=1}^{T}\left(\theta_{t}-G_{t} \theta_{t-1}\right)^{\prime} \Sigma^{-1}\left(\theta_{t}-G_{t} \theta_{t-1}\right)\right\} \\
& \quad \propto \lambda^{p / 2} \exp \left\{-\frac{\lambda}{2}\left(\theta_{0}-m_{0}\right)^{\prime} \Sigma_{0}^{-1}\left(\theta_{0}-m_{0}\right)\right\} \lambda^{a_{0} / 2-1}
\end{aligned}
$$

$$
\cdot \exp \left\{-\frac{b_{0}}{2} \lambda\right\} g(\zeta)
$$

Hence, the conditional posterior density function of $\zeta$ is given as

$$
\begin{aligned}
f^{*} & \left(\zeta \mid \underset{\sim}{\underset{\sim}{y}}\left\{\theta_{t}\right\}_{t=0}^{T}, \lambda\right) \propto \frac{1}{\{\psi(\zeta)\}^{T}} \\
& \cdot \exp \left\{-\frac{\lambda}{2 \sigma_{0}^{2} \psi^{2}(\zeta)} \sum_{t=1}^{T}\left(y_{t}-x_{t}^{\prime} \theta_{t}\right)^{2}\right\} g(\zeta) .
\end{aligned}
$$




\section{An Illustration}

If $\zeta$ follows $\chi^{2}$-distribution with $\nu$ degrees of freedom and $\psi(\zeta)=(\zeta / \nu)^{-(1 / 2)}, \nu>0$, then

$$
\begin{aligned}
& f(\underset{\sim}{y} \mid \theta, \lambda) \\
& \quad=\int_{0}^{\infty} \frac{\lambda^{T / 2} \zeta^{T / 2}}{(2 \pi)^{T / 2} \sigma_{0}^{T} \nu^{T / 2}} \exp \left\{-\frac{\lambda \zeta}{2 \sigma_{0}^{2} \nu} \sum_{t=1}^{T}\left(y_{t}-x_{t}^{\prime} \theta_{t}\right)^{2}\right\} \\
& \cdot \frac{1}{2^{\nu / 2} \Gamma(\nu / 2)} \zeta^{\nu / 2-1} e^{-(\zeta / 2)} d \zeta=\frac{\lambda^{T / 2}}{2^{(T+\nu) / 2} \pi^{T / 2} \sigma_{0}^{T} \nu^{T / 2} \Gamma(\nu / 2)} \\
& \cdot \int_{0}^{\infty} \exp \left[-\frac{\zeta}{2}\left\{1+\frac{\lambda}{\sigma_{0}^{2} \nu} \sum_{t=1}^{T}\left(y_{t}-x_{t}^{\prime} \theta_{t}\right)^{2}\right\}\right] \zeta^{(T+\nu) / 2-1} d \zeta \\
& =\frac{\lambda^{T / 2} \Gamma((T+\nu) / 2)}{\pi^{T / 2} \sigma_{0}^{T} \nu^{T / 2} \Gamma(\nu / 2)\left\{1+\left(\lambda / \sigma_{0}^{2} \nu\right) \sum_{t=1}^{T}\left(y_{t}-x_{t}^{\prime} \theta_{t}\right)^{2}\right\}^{((T+\nu) / 2)}}
\end{aligned}
$$

which is the pdf of a multivariate- $t$ distribution. For $v=1$, the distribution reduces to the multivariate Cauchy distribution. For multivariate- $t$ distribution,

$b_{t}(\zeta)$

$$
= \begin{cases}G_{1}^{\prime} \Sigma^{-1} \theta_{1}+\Sigma_{0}^{-1} m_{0}, & \text { for } t=0 \\ \frac{\zeta}{\sigma_{0}^{2} \nu} x_{t} y_{t}+\Sigma^{-1} G_{t} \theta_{t-1}+G_{t+1}^{\prime} \Sigma^{-1} \theta_{t+1}, & \text { for } t=1,2, \ldots, T-1 \\ \frac{\zeta}{\sigma_{0}^{2} \nu} x_{T} y_{T}+\Sigma^{-1} G_{T} \theta_{T-1}, & \text { for } t=T,\end{cases}
$$

$B_{t}(\zeta)^{-1}$

$$
= \begin{cases}\Sigma_{0}^{\prime}+G_{1}^{\prime} \Sigma^{-1} G_{1}, & \text { for } t=0 \\ \frac{\zeta}{\sigma_{0}^{2} \nu} x_{t} x_{t}^{\prime}+\Sigma^{-1}+G_{t+1}^{\prime} \Sigma^{-1} G_{t+1}, & \text { for } t=1,2, \ldots T-1 \\ \frac{\zeta}{\sigma_{0}^{2} \nu} x_{T} x_{T}^{\prime}+\Sigma^{-1}, & \text { for } t=T .\end{cases}
$$

Further, the conditional posterior density of $\theta_{s}$ given $\left\{\theta^{(s)}, \lambda, \zeta\right\}$ is $N_{p}\left(B_{s}(\zeta) b_{s}(\zeta), \lambda^{-1} B_{s}(\zeta)\right)$ and conditional posterior density of $\lambda$ is gamma. Further,

$$
\begin{aligned}
& \int_{0}^{\infty} \frac{\zeta^{T / 2}}{\nu^{T / 2}} \exp \left\{-\frac{\lambda \zeta}{2 \sigma_{0}^{2} \nu} \sum_{t=1}^{T}\left(y_{t}-x_{t}^{\prime} \theta_{t}\right)^{2}\right\} \frac{1}{2^{\nu / 2} \Gamma(\nu / 2)} \\
& \cdot \zeta^{\nu / 2-1} e^{-(\zeta / 2)} d \zeta \\
& =\frac{2^{T / 2} \Gamma((T+\nu) / 2)}{\nu^{T / 2} \Gamma(\nu / 2)\left\{1+\left(\lambda / \sigma_{0}^{2} \nu\right) \sum_{t=1}^{T}\left(y_{t}-x_{t}^{\prime} \theta_{t}\right)^{2}\right\}^{(T+\nu) / 2}} .
\end{aligned}
$$

Hence, the conditional posterior density of $\zeta$ is given by

$$
\begin{aligned}
& f^{*}\left(\zeta \mid \underset{\sim}{y},\left\{\theta_{t}\right\}_{t=0}^{T}, \lambda\right) \\
& =\frac{\zeta^{(T+v) / 2-1}\left\{1+\left(\lambda / \sigma_{0}^{2} \nu\right) \sum_{t=1}^{T}\left(y_{t}-x_{t}^{\prime} \theta_{t}\right)^{2}\right\}^{(T+\nu) / 2}}{2^{(T+\nu) / 2} \Gamma((T+\nu) / 2)} \\
& \cdot \exp \left[-\frac{\zeta}{2}\left\{1+\frac{\lambda}{\sigma_{0}^{2} \nu} \sum_{t=1}^{T}\left(y_{t}-x_{t}^{\prime} \theta_{t}\right)^{2}\right\}\right] \text {. }
\end{aligned}
$$

\section{Implementation of Gibbs Sampler}

Let the generated Gibbs samples be denoted by $\left(\left\{\theta_{t j}^{(k)}\right\}_{t=0}^{T}\right.$, $\left.\lambda_{j}^{(k)}, \zeta_{j}^{(k)}\right), j=1,2, \ldots, N$. Then by employing Gibbs sampler posterior density of $\theta_{s}$ given $y$ can be estimated as

$$
\begin{aligned}
& \widehat{f}^{*}\left(\theta_{s} \mid \underset{\sim}{y}\right) \\
& =\frac{1}{N} \sum_{j=1}^{N} f^{*}\left(\theta_{s} \mid \theta_{s-1, j}^{(k)}, \theta_{s+1, j}^{(k)}, \lambda_{j}^{(k)}, \zeta_{j}^{(k)}, \underset{\sim}{y}\right), \\
& \text { for } s=0,1, \ldots, T .
\end{aligned}
$$

Recall that the estimated posterior density of $\theta_{s}$ depends on the two values adjacent to $\theta_{s}$. Hence, an estimate of $\theta_{s \mid T}$ is the mean of the estimated density obtained as $\bar{\theta}_{s \mid T}=(1 / N) \sum_{j=1}^{N} B_{s j}^{(k)} b_{s j}^{(k)}$, where $k$ is the number of iterations during implementation of the Gibbs sampler and $b_{s j}^{(k)}$ and $B_{s j}^{(k)}$ are the values of $b(\zeta)$ and $B(\zeta)$ based on $\left(\left\{\theta_{t j}^{(k)}\right\}_{j=1}^{N}, t=s-1, s+1, \zeta_{j}^{(k)}\right)$. Then the fitted value of $y_{t}$ for our model is $\tilde{y}_{t}=x_{t}^{\prime} \bar{\theta}_{t \mid T}$. If one is interested in one step ahead prediction of the unknown value $y_{T+1}$, the Gibbs algorithm can be employed with the following modification. We consider $\left\{\theta_{T+1}, y_{T+1}\right\}$ as an additional parameter to the Gibbs sampler. The conditional density of $y_{T+1}$ is given by $f\left(y_{T+1} \mid\left\{\theta_{t}\right\}_{t=1}^{T+1}, \lambda, \zeta, y\right)=\left(\sqrt{\lambda} / \sqrt{2 \pi} \psi(\zeta) \sigma_{0}\right) \exp [-(\lambda /$ $\left.\left.2 \psi^{2}(\zeta) \sigma_{0}^{2}\right)\left(y_{T+1}-x_{T+1}^{\prime} \theta_{T+1}\right)^{2}\right]$. Then Gibbs sampler can be run to find the following estimates of the marginal densities of $\theta_{T+1}$ and predictive density of $y_{T+1}$, respectively:

$$
\begin{aligned}
& \hat{f}\left(\theta_{T+1} \mid \underset{\sim}{y}\right) \\
& =\frac{1}{N} \sum_{j=1}^{N} f^{*}\left(\theta_{T+1} \mid\left\{\theta_{t j}^{(k)}\right\}_{t=0}^{T}, \lambda_{j}^{(k)}, \zeta_{j}^{(k)}, \underset{\sim}{\underset{\sim}{y}} y_{T+1, j}^{(k)}\right) \\
& \widehat{f}\left(y_{T+1} \mid \underset{\sim}{y}\right) \\
& =\frac{1}{N} \sum_{j=1}^{N} f^{*}\left(y_{T+1} \mid\left\{\theta_{t j}^{(k)}\right\}_{t=0}^{T+1}, \lambda_{j}^{(k)}, \zeta_{j}^{(k)}, \underset{\sim}{y}\right) .
\end{aligned}
$$

Under the squared error loss, $y_{T+1}$ can be predicted by the mean of the estimate of predictive density $\widehat{f}\left(y_{T+1} \mid y\right)$, which is given by

$$
\bar{y}_{T+1}=x_{T+1}^{\prime}\left(\frac{1}{N} \sum_{j=1}^{N} \theta_{T+1, j}^{(k)}\right) .
$$

Alternatively, one step ahead prediction via Gibbs sampler $\left\{y_{T+1, j}^{(k)}\right\}_{j=1}^{N}$ is given by $\bar{y}_{T+1}=(1 / N) \sum_{j=1}^{N} y_{T+1, j}^{(k)}$. The above procedure can be adopted for predicting $y_{T+l} ; l \geq 1$. 


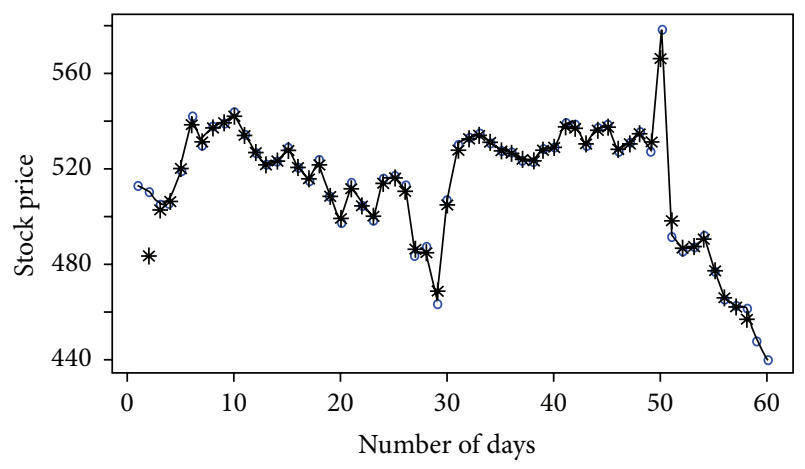

FIGURE 1: Opening price data (represented by hollow circles) and data fitted through Kalman filter model (represented by *). Original data points are joined by straight lines.

\section{Empirical Analysis}

Stock price changes over a 3-month period are studied by analyzing records from database (of yahoo finance) documenting stocks of Amazon.com-an electronic commerce company. The data consists of daily latest intraday delayed opening prices, quoted in US dollars, taken between 6 July, 2015, and 2 October, 2015. The early diagnostics reflect that the data follows the random walk plus noise model which is an elementary form of state space model involving just one state variable. Specification of $x_{t}^{\prime}$ and $G_{t}$ in the model and knowledge of variances and covariances of the disturbance term are a prerequisite for implementing state space models. Based on the external facts and a preliminary examination of the data, we assumed that $p=1, m_{0}=1, a_{0}=2, b_{0}=2$, $\sigma_{0}=1$ (a restrictive assumption), $\Sigma_{0}=\Sigma=1$ (restrictive assumption), $\zeta \sim \chi^{2}(1), x_{t}^{\prime}=1, G_{t}=1$. Analysis for model fitting is based on the first 60 observations. Subsequent 5 observations are excluded from the analysis and are used further in the predictive analysis. We then executed the Gibbs sampler for $k=40$ iterations, using $N=1500$ parallel replications per iteration. A few initial iteration values in the chain were discarded to allow for dissipation of the initialized values called the burn-in values. The Kalman filter is fitted using $\hat{y}_{t}=x_{t}^{\prime}\left((1 / N) \sum_{j=1}^{N} \theta_{t, j}^{(k)}\right)$. The longitudinal profile of the original 60 data points superimposed with the graph of Kalman filter model (via Gibbs sampler) is plotted in Figure 1.

For implementing one step ahead prediction of the observations $y_{r}, r=1, \ldots, 5$, we added $\left\{y_{t+r}, \theta_{t+r}\right\}$ to the Gibbs sampler as described in Section 5. Sample values were generated for $y_{t+r}, r=1, \ldots, 5$, for several parallel chains. $N=$ 1500 replications were conducted in order to obtain smoother estimates. It is observed that the first predicted value is closest to the true value (Table 1). This may be attributed to the fact that the Kalman filter for prediction is based only on the last observed value. Subsequent chains are based on the generated predictions which are subsequently treated as observations. It appears that Gibbs sampler provides a good one step ahead prediction only. The impediment may be overcome by using more efficient computing machines which allow for longer iterative chains and higher quantum of replications.
TABLE 1: Prediction of the opening stock prices up till 5 steps ahead of the recorded data.

\begin{tabular}{lcc}
\hline Observations & True value & Predicted value \\
\hline$Y_{61}$ & 434.9 & 435.2 \\
$Y_{62}$ & 434.35 & 438.26 \\
$Y_{63}$ & 435.68 & 437 \\
$Y_{64}$ & 435.23 & 439.8 \\
$Y_{65}$ & 437 & 445.1 \\
\hline
\end{tabular}

The increased simulation would allow to assess the required length of path which the chain traverses before stabilization occurs for predicting future values and to identify whether more distant predictions could be improved by increasing the number of iterations and/or the length of the chains, since it is known that the predictive power of Gibbs sampler is limited by the choices of the number of iterations, number of replications (parallel chains), and the initial values of the chains [21]. However, the present empirical analysis demonstrates the ability of the Gibbs sampler to provide a good fit to the Kalman filter model for the stock price data modeled using the state space model assuming a single state although the Gibbs algorithm fails to provide satisfactory predictions of the future observations for the volatile stock prices data.

\section{Conclusion}

The present work focuses on the discrete time formulation for the state space models, which are a particular form of stochastic coefficients models and have inherent facility for adapting to model departures through state equations. The objective of developing various time series or econometric models is to evaluate the probability mechanism generating a time series or a set of time series. To understand the global aggregates or the macroquantities such as GNP, CPI, level of employment, production in industry, price or volume of a crop, the amount and types of organic pollutants, or functioning of any other system, the need is to build a theoretical model in order to enable the practitioner to take action or to test the theories generating the observations. Development of the present theoretic framework from the Bayesian perspective is expected to provide a new insight and an enhanced dimension in predictive analysis for applied researchers and practitioners.

\section{Conflict of Interests}

The author declares that there is no conflict of interests regarding the publication of this paper.

\section{Acknowledgments}

The author acknowledges with thanks the computational assistance using R software provided by Ms. Ishna Kaul, Delhi Technical University. The R\&D support grant by University 
of Delhi and DU DST PURSE grant are gratefully acknowledged.

\section{References}

[1] M. G. Kendall, "The analysis of economic time series, part I: prices," Journal of the Royal Statistical Society, Series A, vol. 96, pp. 11-25, 1953.

[2] E. F. Fama, "The behavior of stock-market prices," The Journal of Business, vol. 38, no. 1, pp. 34-105, 1965.

[3] P. D. Praetz, "The distribution of share price changes," The Journal of Business, vol. 45, no. 1, pp. 49-55, 1972.

[4] A. Zellner, "On Bayesian regression analysis with g-prior distributions," Tech. Rep., H.G.B. Alexander Research Foundation, Graduate School of Business, University of Chicago, 1980.

[5] D. A. S. Fraser, Inference and Linear Models, McGraw-Hill, New York, NY, USA, 1979.

[6] B. C. Sutradhar and M. M. Ali, "Estimation of the parameters of a regression model with a multivariate t error variable," Communications in Statistics-Theory and Methods, vol. 15, no. 2, pp. 429-450, 1986.

[7] M. S. Haq and S. Khan, "Prediction distribution for a linear regression model with multivariate student-t error distribution," Communications in Statistics. Theory and Methods, vol. 19, no. 12, pp. 4705-4712, 1990.

[8] S. Khan and M. S. Haq, "Predictive inference for the multilinear model with errors having multivariate $t$-distribution and first order auto-correlation structure," Sankhyā Series B, vol. 56, no. 1, pp. 95-106, 1994.

[9] S. Chib, J. Osiewalski, and M. F. J. Steel, "Posterior inference on the degree of freedom parameter in multivariate- $t$ regression models," Economics Letters, vol. 37, no. 4, pp. 391-397, 1991.

[10] I. R. Prucha and H. H. Kelejian, "The structure of simultaneous equation estimators: a generalization towards nonnormal disturbances," Econometrica, vol. 52, no. 3, pp. 721-736, 1984.

[11] S. Chib, S. R. Jammalamadaka, and R. C. Tiwari, Bayes and Likelihood Prediction in Regressions with Homoscedastic and Heteroscedastic Multivariate-Terrors, Mimeo, New York, NY, USA, 1986.

[12] S. R. Jammalamadaka, R. C. Tiwari, and S. Chib, "Bayes prediction in the linear model with spherically symmetric errors," Economics Letters, vol. 24, no. 1, pp. 39-44, 1987.

[13] D. Kelker, "Distribution theory of spherical distributions and a location-scale parameter generalization," Sankhyā. Series A, vol. 32, pp. 419-430, 1970.

[14] M. A. Chmielewski, "Elliptically symmetric distributions: a review and bibliography," International Statistical Review, vol. 49, no. 1, pp. 67-76, 1981.

[15] T. Klock and H. K. Van Dijk, "Bayesian estimates of equation systems parameters: an application of integration by Monte Carlo," Econometrica, vol. 46, pp. 1-20, 1978.

[16] J. C. Naylor and A. F. M. Smith, "Econometric illustrations of novel numerical integration strategies for Bayesian inference," Journal of Econometrics, vol. 38, no. 1-2, pp. 103-125, 1988.

[17] J. Geweke, "Antithetic acceleration of Monte Carlo integration in Bayesian inference," Journal of Econometrics, vol. 38, no. 1-2, pp. 73-89, 1988.

[18] J. Geweke, "Bayesian inference in econometric models using Monte Carlo integration," Econometrica. Journal of the Econometric Society, vol. 57, no. 6, pp. 1317-1339, 1989.
[19] J. Geweke, "Evaluating the accuracy of sampling-based approaches to the calcuation of posterior moments," Research Department Staff Report 148, Federal Reserve Bank of Minneapolis, Minneapolis, Minn, USA, 1991.

[20] R. C. Tiwari, Y. Yang, and J. N. Zalkikar, "Time series analysis of BOD data using the Gibbs sampler," Environmetrics, vol. 7, no. 6, pp. 567-578, 1996.

[21] M. K. Cowles and B. P. Carlin, "Markov chain Monte Carlo convergence diagnostics: a comparative review," Journal of the American Statistical Association, vol. 91, no. 434, pp. 883-904, 1996. 


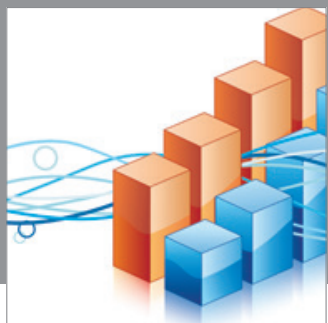

Advances in

Operations Research

mansans

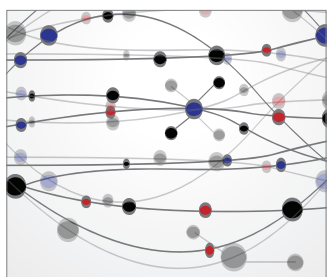

The Scientific World Journal
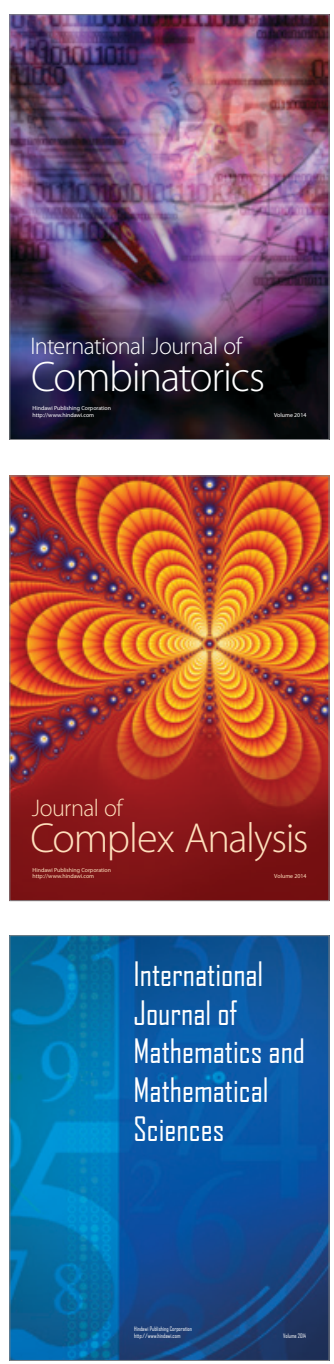
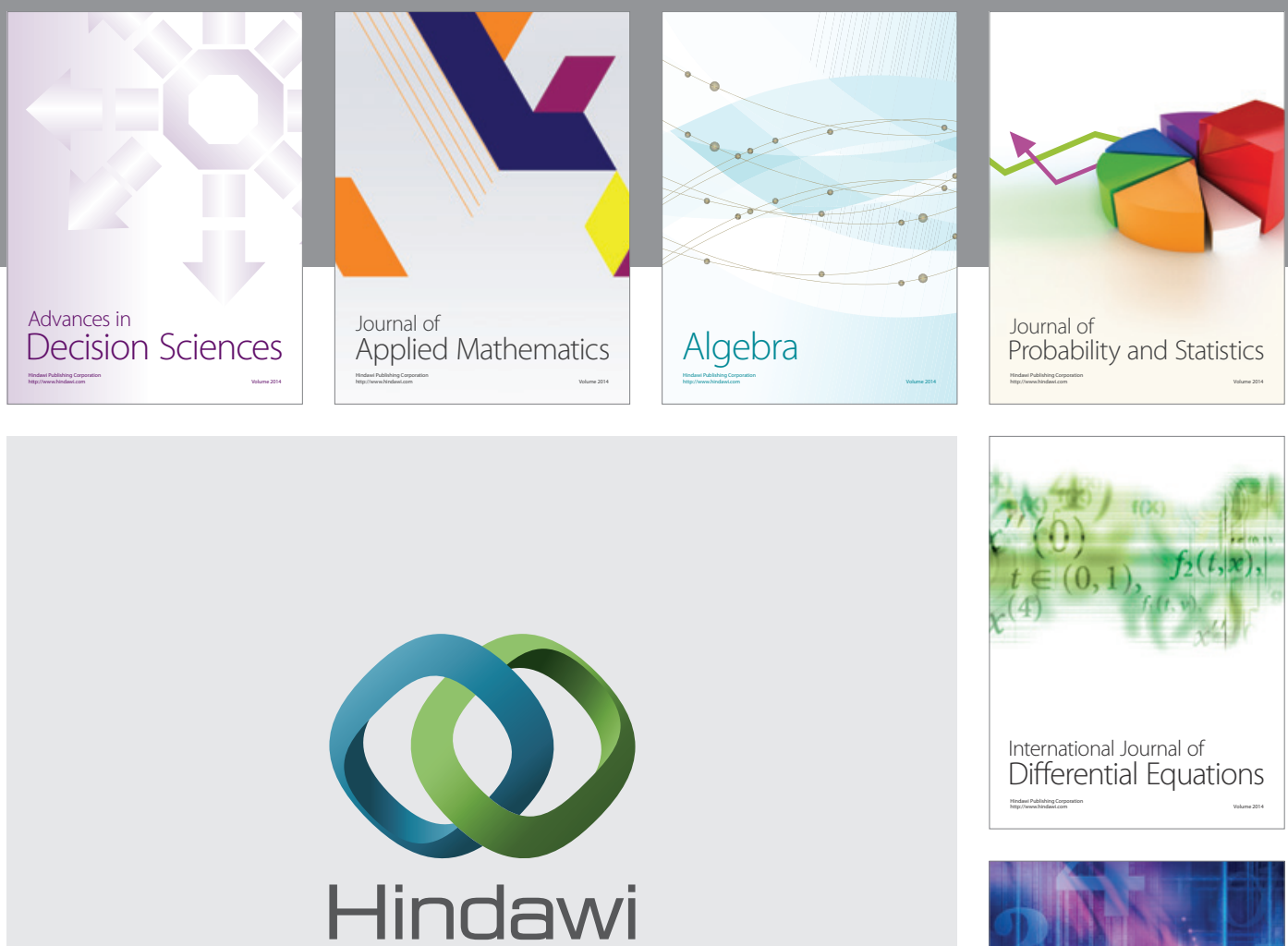

Submit your manuscripts at http://www.hindawi.com
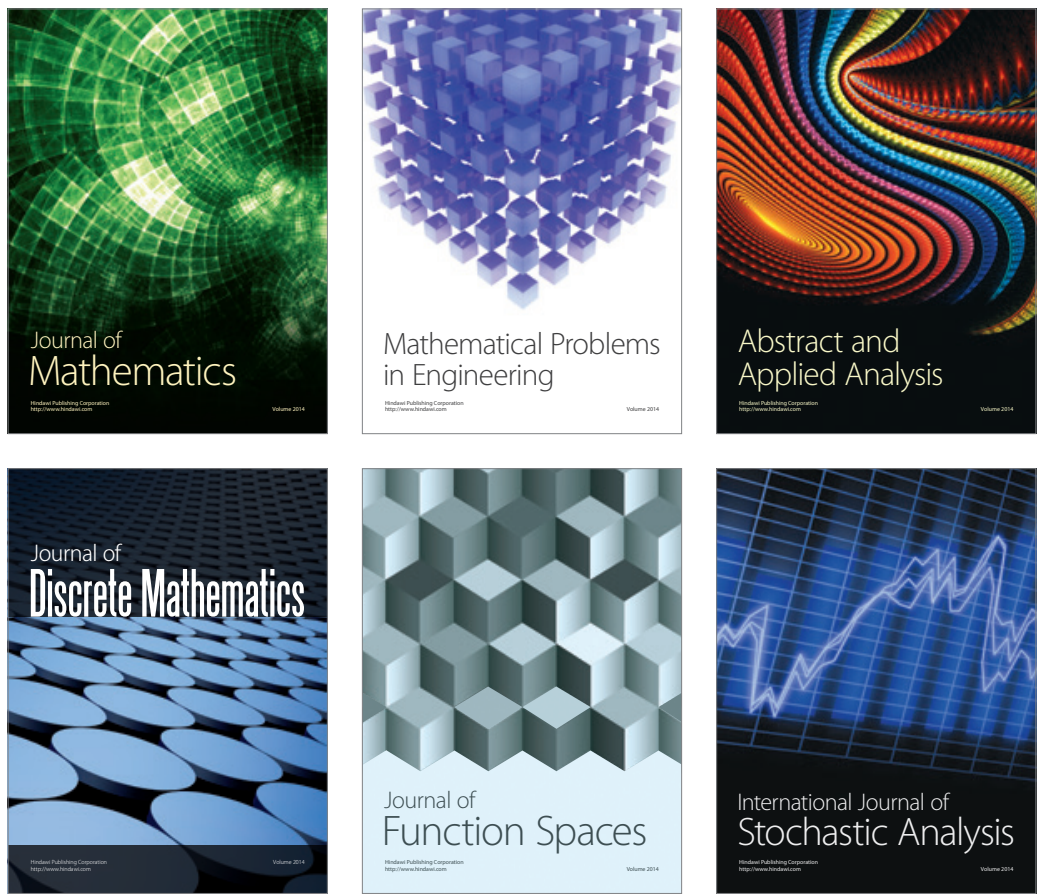

Journal of

Function Spaces

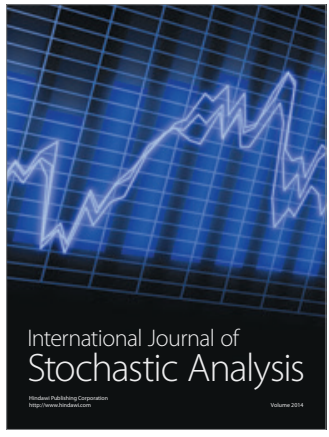

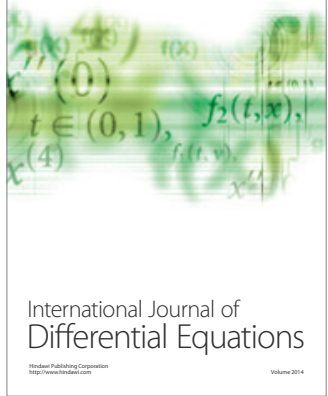
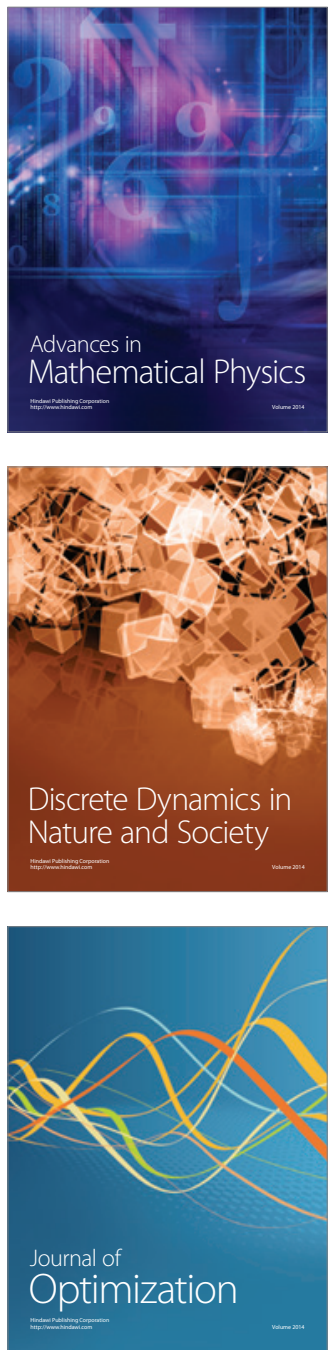